\title{
Atoms Absorb Low Frequency Electromagnetic Energy
}

\author{
Hans W. Giertz \\ Uppsa Research, Gnesta, Sweden \\ Email: hans@miklagaard.com
}

Received August 16, 2013; revised September 16, 2013; accepted September 23, 2013

Copyright (C) 2013 Hans W. Giertz. This is an open access article distributed under the Creative Commons Attribution License, which permits unrestricted use, distribution, and reproduction in any medium, provided the original work is properly cited.

\begin{abstract}
This paper presents a method to measure TEM (transverse electromagnetic) waves absorbed by atoms. The results show the existence of a singularity in the universe which generates a broad spectrum of synchronized and extremely low frequency TEM waves in the period range $30-160 \mathrm{~s}$. Atoms create resonance and absorb these TEM waves. The absorbed energy consists of a set of TEM waves where the sum generates an intrinsic static electric and magnet field. It is proposed that this static electric and magnetic field induces static forces within the atomic nucleus. The study describes a method to measure TEM waves absorbed by atoms (direction, frequency and amplitude). The study displays that chemical elements absorb TEM waves of different periods, supporting the hypothesis that TEM waves are absorbed by an atomic intrinsic resonance mechanism. The study displays a non-invasive method facilitating novel analyses of the atomic nucleus.
\end{abstract}

Keywords: Atom; Nucleus; Elementary Particle; Strong Force; Gluon; TEM Wave; Singularity

\section{Introduction}

The atomic structure is described by the Standard Model [1]. Despite huge scientific efforts the atomic structure remains to large extent unsolved. All forces within the atom, except the Coulomb force, remain unknown; the strong force (gluon), the weak force and the graviton [2-5]. Some progress has been achieved in finding the Higgs boson [6]. Some scientists have brought forward a rather controversial model. They claim that the universe contains an omnipresent form of energy called dark energy [7], field, zero-point-fluctuation or zero-point energy [8]. It has been proposed that gravity is caused by this energy, and the energy creates mass and atomic forces $[9,10]$. The drawback with these studies is that they are theoretical and not based on empiric results and they lack in depth description of the omnipresent field and its energy.

According to the Standard Model atomic forces and energy are created by the atom itself, i.e. the atom and its processes are self-contained. Recently published papers show that this is not always the case $[11,12]$. It has been reported that the universe contains a gigantic singularity that emits enormous amount of TEM (transverse electromagnetic) waves with frequencies $69.9 \mathrm{~Hz}$ and 91.9 $\mathrm{Hz}$ [11]. These TEM waves create resonance with atoms and are then re-emitted without change of content. This creates a mutual flow of synchronized TEM waves between atoms and where the energy is described by the Poynting vector. The mutual flow of energy between atoms creates mutual force of attraction, i.e. gravity. These TEM waves are called gravity waves. It has been proposed that the atomic intrinsic resonance mechanism consists of configurations of positive and negative elementary electric charges $[11,12]$.

Furthermore, it has been reported that photons are packages of EM (electromagnetic) energy (i.e. $h v$ ) superpositioned on the above TEM waves or gravity waves [12]. The function is as follows, illustrated with a photon generated by a decelerated electron. The electron's configurations of charges create resonance with gravity waves with frequency $69.9 \mathrm{~Hz}$ and/or $91.9 \mathrm{~Hz}$. The electron is decelerated and the excess energy $h v$ is then superpositioned on a gravity wave which then has the instant frequency $h v+69.9 \mathrm{~Hz}$ or $h v+91.9 \mathrm{~Hz}$. Hence, gravity waves or TEM waves originating from the singularity are carriers of EM energy in space.

TEM waves of extremely low frequency are impossible to detect or to measure using state of the art methods and instruments available within science. This explains why this energy is unknown. However, innovative technique makes it possible to measure these TEM waves [13]. Hence, it is possible to measure TEM waves emit- 
ted by the singularity, as described previously $[11,12]$. The present study describes how TEM waves absorbed by atoms are measured. In the present study the singularity is called the Energy Source.

The present study displays that the Energy Source emits extremely low frequency TEM waves with periods $T=30-160 \mathrm{~s}$. Furthermore, the study displays that atoms create resonance with these TEM waves and absorb a part of the spectrum. The absorbed spectrum creates, according to Fourier synthesis [14], static electric and magnetic fields within the atom. Static electric and magnetic fields which operate on atomic intrinsic charges may create internal forces. Hence, some atomic forces may not have intrinsic origin, however, consist of induced forces created by external energy which originates from a singularity in the universe. It is proposed that these processes follow the laws of classical electromagnetic theory and wave theory [15-19].

The present study is structured as follows. Section 2 describes materials and methods which facilitated measurement of TEM waves emitted from the Energy Source. Measurement of Energy Source TEM waves were performed as described in [11-13]. A method is described which facilitated measurement of the atom's TEM wave absorption spectrum. Section 3 encompasses results. The chemical elements $\mathrm{Ag}, \mathrm{Au}, \mathrm{C}, \mathrm{Cl}, \mathrm{Fe}, \mathrm{Hg}, \mathrm{Na}, \mathrm{P}, \mathrm{Pb}$ and $\mathrm{S}$ were measured with respect to their absorbed TEM wave spectrum. Section 4 covers discussion and conclusions.

The aim of the study is to characterize the atom's TEM wave absorption spectrum in the period range $T=$ $30-160 \mathrm{~s}$.

\section{Materials and Methods}

All measurements in the present study encompassed TEM waves in the period range $T=30-160 \mathrm{~s}$. It is apparently impossible to detect TEM waves with such enormous periods and wavelengths $\left(\approx 3 \times 10^{10} \mathrm{~m}\right)$. In the present study these TEM waves were not regarded as waves; however, as quasi static, polarized electric and magnetic fields. Hence, measurements were reduced to identifying quasi static electric and magnetic fields, i.e. the TEM wave's electric and magnetic field vectors.

TEM waves absorbed by atoms (a chemical element) originate from a singularity in the universe in direction beyond the Milky Way galactic centre. The direction is towards north, along the earth's rotational axis; however, misaligned by approximately 10 degrees, as described previously [11]. Hence, the TEM wave direction followed a diurnal, counter-clockwise circle with approximately 20 degrees cone angel. Its peak altitude was approximately 75 degrees and occurred at noon (in Sweden) and the direction was towards the geographical north $+/-$ 5 degrees (see Figure 1). The magnetic field vectors

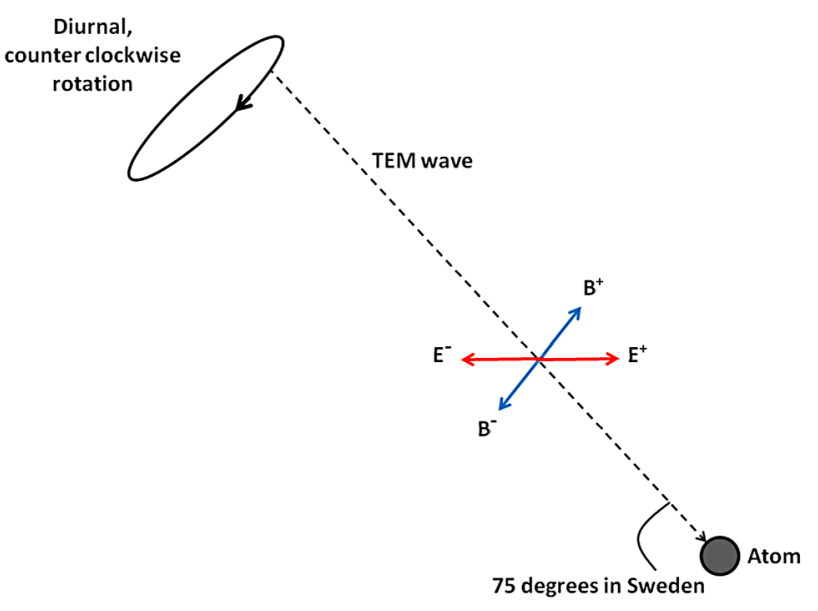

Figure 1. The atom absorbed TEM waves originating from a source towards north and aligned approximately along the earth's rotational axis. The TEM waves were polarized and the electric field vectors were always oriented in the horizontal plane.

were always directed in the vertical plane, caused by influence from the geomagnetic field. Thus, the electric field vectors were always directed in the horizontal plane, i.e. all TEM waves were polarized. Hence, the field vectors had always a fixed position which was easy to identify. Measurements were always performed by measuring the peak amplitude of the electric field vector $\boldsymbol{E}^{+}$as a function of time. This procedure demanded patience and practice in order to separate TEM waves with different periods.

Measurements of plane TEM wave field vector amplitudes were made as described in previous papers [11-13]. Those papers describe in detail how the position and amplitude of electric and magnetic field vectors were measured using a charge meter. The charge meter has been described in detail previously [13]. In summary the charge meter contained a probe with charge density $\rho$. The probe was moved, with constant speed, through the TEM wave electric field vectors $\boldsymbol{E}$. This resulted in an electric body force $\rho \boldsymbol{E}$ on the probe charge density $\rho$.

This current pulse was amplified and displayed. In this case $\boldsymbol{E}$ was field vectors, implying that $\operatorname{div} \boldsymbol{E}$ was large and resulted in a distinct current pulse. This probe was also moved, with constant speed, through the TEM magnetic field vectors $\boldsymbol{B}$, which resulted in a magnetic body force $\boldsymbol{J} \times \boldsymbol{B}$ on current $\boldsymbol{J}$ in the probe. In this case $\boldsymbol{B}$ was field vectors, implying that $\operatorname{div} \boldsymbol{B}$ was large and resulted in a distinct current pulse. The measurement method had one important feature. The field vector's physical length was proportional to its amplitude and in the present study between $1-10 \mathrm{~m}$. It was easy to measure and determine the physical length of the field vectors since $\operatorname{div} \boldsymbol{E}$ and $\operatorname{div} \boldsymbol{B}$ were large. Hence, this was an accurate measure of the relative amplitude. 
Measurement of chemical elements was performed by dissolving an extremely small amount of the chemical element ions in de-ionized water. The solution was positioned in a small glass $(\mathrm{Si})$ container. The natural periods of $\mathrm{H}, \mathrm{O}$ and $\mathrm{Si}$ deviated from the natural periods of the chemical elements (Ag, Au, C, Cl, Fe, Hg, Na, P, Pb and $\mathrm{S}$ ) included in the study. Thus, it was possible to filter the influence from $\mathrm{H}_{2} \mathrm{O}$ and $\mathrm{Si}$.

Measurements were performed $50 \mathrm{~km}$ south of Stockholm, Sweden.

Analysis: The experiments were performed blindly, randomly and repeated three times.

\section{Results}

Experiment 1: The purpose with this experiment was to measure the period of TEM waves absorbed by the chemical elements $\mathrm{Ag}, \mathrm{Au}, \mathrm{C}, \mathrm{Cl}, \mathrm{Fe}, \mathrm{Hg}, \mathrm{Na}, \mathrm{P}, \mathrm{Pb}$ and $\mathrm{S}$ in the period range $T=30-160 \mathrm{~s}$. One chemical element was dissolved as ions in extremely pure and de-ionized water (Milli-Q). Subsequently $1 \mathrm{ml}$ solution was mixed with $999 \mathrm{ml}$ or $9 \mathrm{ml}$ water, thereby reducing the concentration of ions by a factor of thousand or ten. This procedure was repeated until the solution resulted in a field vector length of approximately $1-10 \mathrm{~m}$ at the measured period $T$. Au was measured by rubbing an extremely small amount of $\mathrm{Au}$ on the glass container surface. This enabled measurement of field vector lengths (amplitudes) of the absorbed TEM wave spectrums and natural periods of $\mathrm{Ag}, \mathrm{Au}, \mathrm{C}, \mathrm{Cl}, \mathrm{Fe}, \mathrm{Hg}, \mathrm{Na}, \mathrm{P}, \mathrm{Pb}$ and $\mathrm{S}$. Known solutions of $\mathrm{P}$ were used $(17.8 \mathrm{mg} / 1,32.6 \mathrm{mg} / \mathrm{l}$ and $54 \mathrm{mg} / \mathrm{l}$ ) in order to calibrate measurements. It was estimated that approximately $0.1 \mathrm{fg}\left(10^{-16} \mathrm{~g}\right)$ of $\mathrm{P}$ resulted in $10 \mathrm{~m}$ field vector length at $T=41 \mathrm{~s}$. It was found that $\mathrm{Ag}, \mathrm{Cl}, \mathrm{Pb}$ and $\mathrm{Na}$ received TEM waves with the approximate period $T=120 \mathrm{~s}$ and its even harmonics $T_{2}=60 \mathrm{~s}$ and $T_{4}=30 \mathrm{~s}$. However, each harmonic consisted of many TEM waves where the periods deviated approximately $1 \mathrm{~s}$, e.g. $T=119 \mathrm{~s}, T=120 \mathrm{~s}, T=121 \mathrm{~s}$ and $T=123$ s. Hence, $\mathrm{Ag}, \mathrm{Cl}, \mathrm{Pb}$ and $\mathrm{Na}$ created resonance and absorbed TEM waves with slightly different periods. It was found that $\mathrm{Au}, \mathrm{C}, \mathrm{Fe}, \mathrm{Hg}, \mathrm{P}$ and $\mathrm{S}$ absorbed TEM waves with the approximate period $T=160$ $\mathrm{s}$ and its even harmonics $T_{2}=80 \mathrm{~s}$ and $T_{4}=40 \mathrm{~s}$. However, each harmonic consisted of many TEM waves where the periods deviated approximately $1 \mathrm{~s}$, e.g. $T=$ $159 \mathrm{~s}, T=160 \mathrm{~s}$ and $T=161 \mathrm{~s}$. Hence, Au, C, Fe, Hg, P and $\mathrm{S}$ created resonance and absorbed TEM waves with slightly different periods. The results are displayed in Table 1.

Experiment 2: The purpose with this experiment was to identify if chemical elements absorbed static electric and magnetic fields caused by the sum of many TEM waves (according to Fourier synthesis [14]). Experiment 1 was repeated. However, this time the static field ab-
Table 1. Periods $T$ of TEM waves absorbed by the chemical elements Ag, Au, C, Cl, Fe, Hg, Na, P, Pb and S.

\begin{tabular}{cccc}
\hline Chemical element & $T(\mathrm{~s})$ & $T_{2}(\mathrm{~s})$ & $T_{4}(\mathrm{~s})$ \\
\hline $\mathrm{Ag}$ & 119 & 59 & 29 \\
$\mathrm{~Pb}$ & 120 & 60 & 30 \\
$\mathrm{Na}$ & 121 & 61 & 31 \\
$\mathrm{Cl}$ & 123 & 63 & 33 \\
$\mathrm{Fe}$ & 159 & 79 & 39 \\
$\mathrm{C}$ & 160 & 80 & 40 \\
$\mathrm{P}$ & 161 & 81 & 41 \\
$\mathrm{~S}$ & 161 & 81 & 41 \\
$\mathrm{Hg}$ & 163 & 83 & 43 \\
$\mathrm{Au}$ & 165 & 85 & 45 \\
\hline
\end{tabular}

sorbed by $\mathrm{Ag}, \mathrm{Au}, \mathrm{C}, \mathrm{Cl}, \mathrm{Fe}, \mathrm{Hg}, \mathrm{Na}, \mathrm{P}, \mathrm{Pb}$ and $\mathrm{S}$ was measured. It was observed that chlorine $\mathrm{Cl}$ absorbed a static field consisting of static electric and static magnetic field vectors. The field vector length was measured as a function of the time. It displayed that the field vector length was constant and with a superpositioned field variation of $+/-5 \%$, see Figure 2. Measuring the variation of the superpositioned field as a function of time allowed an accurate measure of the superpositioned pe$\operatorname{riod} T_{\mathrm{s}}$, and for $\mathrm{Cl}, T_{\mathrm{s}}=33 \mathrm{~s}$. The procedure was repeated to include all chemical elements and their periods in the 30 - 160 s range listed in Table 2. This displayed that all chemical elements exhibited similar behavior, i.e. a static field and a small superpositioned field. However, the relative amplitude of the superpositioned field as well as its pattern and period varied between the measured chemical elements. The period of the superpositioned field for $\mathrm{Ag}, \mathrm{Au}, \mathrm{C}, \mathrm{Cl}, \mathrm{Fe}, \mathrm{Hg}, \mathrm{Na}, \mathrm{P}, \mathrm{Pb}$ and $\mathrm{S}$ is displayed in Table 2. Figure 2 also shows the TEM wave amplitude of absorbed energy, in $\mathrm{Cl}$, at its natural periods $T=123 \mathrm{~s}$, $T_{2}=63 \mathrm{~s}$ and $T_{4}=33 \mathrm{~s}$, as described in Experiment 1, Table 1. For chlorine $\mathrm{Cl}$ the peak amplitude, at the natural period $T=33 \mathrm{~s}$, was $85 \%$ of the static field vector amplitude.

Experiment 3: The purpose with this experiment was to investigate the accuracy of the atomic resonance mechanism. The background is that TEM waves add linearly when they have exactly the same period and phase. TEM waves do not add when they have different periods and when these periods and their phases do not create interference. TEM waves can also create mutual interference. Two samples of chlorine $\mathrm{Cl}$ were prepared. The amplitudes (as a function of time) of absorbed TEM waves at natural periods $T, T_{2}$ and $T_{4}$ as well as the static field and the superpositioned field (as displayed in Figure 2) were measured for each sample of $\mathrm{Cl}$. Subsequently the two samples of $\mathrm{Cl}$ were positioned side by side and the amplitudes (as a function of time) of TEM waves at the natural periods $T, T_{2}$ and $T_{4}$ as well as the static field and 


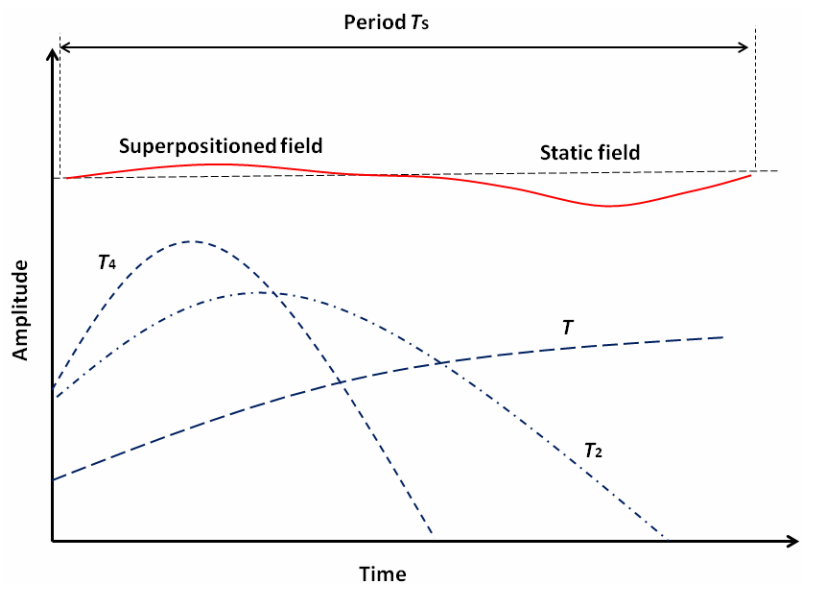

Figure 2. Field vector amplitude of TEM waves absorbed by chlorine $\mathrm{Cl}$. Measurement displayed a static field with a superpositioned field with period $T_{s}=33 \mathrm{~s}$. The figure also displays absorbed TEM waves at the chemical element natural periods $T, T_{2}$ and $T_{4}$ (dotted). The figure is schematic and shows only a few harmonics.

Table 2. Period $T_{\mathrm{s}}$ of the superpositioned field absorbed by the chemical elements $\mathrm{Ag}, \mathrm{Au}, \mathrm{C}, \mathrm{Cl}, \mathrm{Fe}, \mathrm{Hg}, \mathrm{Na}, \mathrm{P}, \mathrm{Pb}$ and S.

\begin{tabular}{cc}
\hline Chemical element & $T_{s}(\mathrm{~s})$ \\
\hline $\mathrm{Ag}$ & 29 \\
$\mathrm{~Pb}$ & 30 \\
$\mathrm{Na}$ & 31 \\
$\mathrm{Cl}$ & 33 \\
$\mathrm{Fe}$ & 39 \\
$\mathrm{C}$ & 40 \\
$\mathrm{P}$ & 41 \\
$\mathrm{~S}$ & 41 \\
$\mathrm{Hg}$ & 43 \\
$\mathrm{Au}$ & 45 \\
\hline
\end{tabular}

the superpositioned field were measured. Results displayed that the measured amplitudes in all cases were equal to the sum of amplitudes of the two samples of $\mathrm{Cl}$. Hence, the electromagnetic processes of the two samples added linearly. This procedure was repeated for $\mathrm{Ag}, \mathrm{Au}$, $\mathrm{C}, \mathrm{Fe}, \mathrm{Hg}, \mathrm{Na}, \mathrm{P}, \mathrm{Pb}$ and $\mathrm{S}$ displaying similar results. Then one sample of $\mathrm{Cl}$ was positioned side by side with one sample of Ag. This time the amplitudes of TEM waves at the natural periods $T, T_{2}$ and $T_{4}$ as well as the static field and the superpositioned field did not add, nor did they interfere. $\mathrm{Ag}$ and $\mathrm{Cl}$ displayed exactly the same amplitudes as when they were measured separately. Hence, the electromagnetic processes of $\mathrm{Ag}$ and $\mathrm{Cl}$ did not influence each other. The experiment was repeated by investigating all combinations of $\mathrm{Ag}, \mathrm{C}, \mathrm{Fe}, \mathrm{Hg}, \mathrm{Na}, \mathrm{P}, \mathrm{Pb}$ and $\mathrm{S}$. Note that $\mathrm{Au}$ is described in Experiment 4. All cases, except one, displayed that there was no influence between the chemical elements. The exception was P and
$\mathrm{S}$; their field vectors at the natural periods $T, T_{2}$ and $T_{4}$ as well as the static field and superpositioned field added linearly when samples of $\mathrm{P}$ and $\mathrm{S}$ were positioned side by side. The reason that $\mathrm{P}$ and $\mathrm{S}$ added linearly was that $\mathrm{P}$ and $\mathrm{S}$ absorbed TEM waves with identical frequencies and phases.

Experiment 4: Experiment 3 was repeated; however, this time one sample of $\mathrm{Au}$ was positioned side by side with one sample of Ag. The two chemical elements did interfere and the static field of $\mathrm{Ag}$ and $\mathrm{Au}$ were each reduced to approximately $10 \%$ of their respectively original static field amplitudes. Subsequently the experiment was repeated by investigating the combination of $\mathrm{Au}$ with $\mathrm{C}, \mathrm{Fe}, \mathrm{Na}, \mathrm{P}, \mathrm{Pb}$ and $\mathrm{S}$. It displayed that $\mathrm{Au}$ did interfere with $\mathrm{C}, \mathrm{Fe}, \mathrm{Na}, \mathrm{P}, \mathrm{Pb}$ and $\mathrm{S}$ whereby the static field of each chemical element was reduced by approximately $90 \%$. Subsequently one sample of Au was positioned side by side with one sample of $\mathrm{Hg}$. This time the amplitudes of TEM waves at the natural periods $T, T_{2}$ and $T_{4}$ of $\mathrm{Au}$ and $\mathrm{Hg}$ as well as the static field of $\mathrm{Au}$ and $\mathrm{Hg}$ did not add, nor did they interfere. Au together with $\mathrm{Hg}$ displayed exactly the same amplitudes as when they were measured separately.

\section{Discussion and Conclusions}

It has been reported that a singularity in the universe generates TEM waves with frequencies $69.9 \mathrm{~Hz}$ and 91.9 $\mathrm{Hz}$ and that atoms absorb and re-emit these TEM waves [11]. The present study displays that a singularity in the same direction generated TEM waves with the period $T=$ $30-160 \mathrm{~s}$. It is reasonable to assume that one and the same singularity generated $69.9 \mathrm{~Hz}$ and $91.9 \mathrm{~Hz}$ and the period $T=30-160 \mathrm{~s}$ (approximately $0.03-0.006 \mathrm{~Hz}$ ).

Experiment 1 displayed that the chemical elements $\mathrm{Ag}$, $\mathrm{Au}, \mathrm{C}, \mathrm{Cl}, \mathrm{Fe}, \mathrm{Hg}, \mathrm{Na}, \mathrm{P}, \mathrm{Pb}$ and $\mathrm{S}$ absorbed subsets of TEM waves in the period range $T=30-160 \mathrm{~s}$. Chemical elements absorbed TEM waves at different periods. The conclusion is that the atomic protons and neutrons or combinations of protons and neutrons had natural periods depending on chemical element type (§ 1). Such absorption of EM energy at discrete periods/frequencies can only be achieved by means of an atomic intrinsic resonance mechanism $(\S 2)$. Resonance with TEM waves can only be achieved by means of a resonance mechanism which contains electric charges $(\S 3)$. From this can be concluded that the atomic nucleus must contain large amount of charges and that these charges must have one or many chemical element specific configurations which create chemical element specific resonance ( $\$ 4)$.

Experiment 2 displayed that every investigated chemical element absorbed a mix of TEM waves with periods which resulted in static electric and magnetic fields (§5). This is in accordance with Fourier synthesis [14]. It is 
reasonable to assume that static electric and magnetic fields which operate on intrinsic atomic charges induce static forces within the atom. Hence, at least one force within the atom may be created by TEM waves originating from a singularity $(\S 6)$. This force can most likely be described based on classical electromagnetic theory and wave theory (§ 7) [15-19].

Experiment 3 displayed the precision of the atomic resonance mechanism. Each of the chemical elements Ag, $\mathrm{Au}, \mathrm{C}, \mathrm{Cl}, \mathrm{Fe}, \mathrm{Hg}, \mathrm{Na}$ and $\mathrm{Pb}$ had its specific resonance mechanism with chemical element specific natural periods, which supports the results in Experiment 1 ( $\$ 1$ ). The results displayed that there was no interference between the chemical elements $\mathrm{Ag}, \mathrm{C}, \mathrm{Cl}, \mathrm{Fe}, \mathrm{Hg}, \mathrm{Na}$ and $\mathrm{Pb}$ and hence, there was no or small overlap of natural periods between these chemical elements. The exception was $\mathrm{P}$ and $\mathrm{S}$ which seemed to have identical or similar resonance mechanisms and natural periods. The conclusion is that the atomic resonance mechanism was extremely accurate and stable $(\S 8)$.

Experiment 4 displayed that $\mathrm{Au}$ created interference with $\mathrm{Ag}, \mathrm{C}, \mathrm{Cl}, \mathrm{Fe}, \mathrm{Na}, \mathrm{P}, \mathrm{Pb}$ and $\mathrm{S}$, whereby the absorbed static electric and magnetic fields of $\mathrm{Ag}, \mathrm{Au}, \mathrm{C}$, $\mathrm{Cl}, \mathrm{Fe}, \mathrm{Na}, \mathrm{P}, \mathrm{Pb}$ and $\mathrm{S}$ were reduced by approximately 90\%. However, $\mathrm{Au}$ and $\mathrm{Hg}$ did not influence each other, i.e. they did not interfere. Gold Au seldom creates compounds; however, Au occurs in alloys with mercury $\mathrm{Hg}$. Maybe compounds and alloys are created by chemical elements that do not interfere $(\S 9)$ ? Experiment 1 displayed that $0.1 \mathrm{fg}$ of a chemical element created electromagnetic fields which were possible to detect at $10 \mathrm{~m}$ distance. Hence, there may be a significant electromagnetic influence between adjacent atoms ( $\$ 10)$.

The conclusion is that atoms created resonance and absorbed electromagnetic energy which originated from a singularity in the universe. The absorbed energy resulted in intrinsic static electric and magnetic fields. Chemical elements created chemical element specific resonance. Combinations of some chemical elements resulted in mutual resonance (e.g. $\mathrm{P}$ and $\mathrm{S}$ ) or no mutual influence (e.g. $\mathrm{Ag}, \mathrm{C}, \mathrm{Cl}, \mathrm{Fe}, \mathrm{Hg}, \mathrm{Na}$ and $\mathrm{Pb}$ ) while other combinations created interference (e.g. Au with Ag, C, Cl, Fe, Na, $\mathrm{P}, \mathrm{Pb}$ and $\mathrm{S})$. It is proposed that the intrinsic static electric and magnetic fields may create static forces between atomic charges and configurations of charges (e.g. within and between protons and neutrons).

The resonance phenomena described in the present paper may seem strange to scientists with background in quantum physics or elementary particle physics. However, the results display that atoms behave according to classical electromagnetic theory and wave theory and where each atom can be described as a set of many forced damped oscillators $[15,16]$. The technique to use forced damped oscillators in order to absorb, radiate, add and mix EM energies and to create interference between EM energies has been utilized within optics and radio technology for more than a century. Light is a TEM wave, the radio wave is the same type a TEM wave, although with lower frequency. The atom absorbs the same type of TEM wave, although with extremely low frequency. The theory of the forced damped oscillator is independent of its frequency. From this can be concluded that the atom absorbs TEM waves by means of its forced damped oscillators, gravity is the result of TEM waves absorbed and re-emitted by atomic forced damped oscillators [11] and photons are generated within atomic forced damped oscillators [12]. Consequently, a substantial part of atomic phenomena are the result of external TEM waves operating on atomic charges configured into forced damped oscillators, according to the laws of electromagnetic and wave theory.

This leads to a question. Do we believe that the atom also contains particles which apply to a fundamentally different and undefined type of physics? Such inconsistency is difficult to explain. Maybe the gluon, the strong and the weak force consist of induced forces created by absorbed TEM waves? Perhaps the electron and the proton are stable forced damped oscillators created by external TEM waves operating on configurations of charges. Splitting the proton may result in particles which are fragments of the proton's configuration and its forced damped oscillators. Hence, the proton's forced damped oscillators are destroyed and cease to absorb TEM waves and cease to induce forces. It results in particle decay. Parts of the proton's configuration may be stable and constitutes stable forced damped oscillators. These particles (e.g. electron) do not decay.

Perhaps the Standard Model can be replaced with a model consisting of only three components. In this model the atom consists of only positive and negative elementary electric charges (i.e. the first component). They are configured into different forced damped oscillators (i.e. the second component) which describe the stable particles, their forces and their energy. A singularity (i.e. the third component) generates TEM waves which are absorbed by the atomic forced damped oscillators and which induce atomic forces and energy. This model applies to the laws of electromagnetism and wave theory.

\section{REFERENCES}

[1] J. F. Donoghue, E. Golowich and B. R. Holstein, "Dynamics of the Standard Model," Cambridge University Press, Cambridge, 1994.

[2] P. Söding, "On the Discovery of the Gluon," European Physical Journal H, Vol. 35, No. 1, 2010, pp. 3-28. http://dx.doi.org/10.1140/epjh/e2010-00002-5

[3] W. Greiner, B. Müller, "Gauge Theory of Weak Interactions," Springer, Berlin, 2009. 
[4] S. Braibant, G. Giacomelli and M. Spurio, "Particles and Fundamental Interactions: An Introduction to Particle Physics," Springer, Berlin, 2009.

[5] W. M. Yao, et al. "Review of Particle Physics," Journal of Physics G; Nuclear and Particle Physics, Vol. 33, No. 1, 2006, pp. 1-94.

http://dx.doi.org/10.1088/0954-3899/33/1/001

[6] V. Khachatryan, A. M. Sirunyan, A.Tumasyan, W. Adam, E. Aguilo, T. Bergauer, M. Dragicevic, et al., "Observation of a New Boson at a Mass of $125 \mathrm{GeV}$ with the CMS Experiment at the LHC," Physics Letters B, Vol. 716, No. 1, 2012, pp. 30-61. http://dx.doi.org/10.1016/j.physletb.2012.08.021

[7] P. J. E. Peebles and B. Ratra, "The cosmological constant and dark energy," Reviews of Modern Physics, Vol. 75. No. 2, 2003, pp. 559-606. http://dx.doi.org/10.1103/RevModPhys.75.559

[8] H. Puthoff, "Ground State of Hydrogen as Zero-PointFluctuation-Determined State," Physical Review D, Vol. 35, No. 10, 1987, pp. 3260-3270.

[9] H. Puthoff, "Source of Vacuum Electromagnetic ZeroPoint Energy," Physical Review A, Vol. 40, No. 9, 1989, pp. 4857-4862.

[10] B. Haish, A. Rueda and D. C. Cole, "Vacuum Zero-Point Field Pressure Instability in Astrophysical Plasma and the Formation of Cosmic Voids," Astrophysical Journal, Vol. 445, 1995, pp. 7-16.

[11] H. W. Giertz, "Gravity Caused by TEM Waves Operating on Dipoles in Atoms," International Journal of Astron- omy and Astrophysics Vol. 3, No. 2A, 2013, pp. 39-50. http://dx.doi.org/10.4236/ijaa2013.32A007

[12] H. W. Giertz, "Photons are EM Energy Superpositioned on TEM Waves," Open Journal of Microphysics, Vol. 3, No. 3, 2013, pp. 71-80. http://dx.doi.org/10.4236/ojm.2013.33013

[13] H. W. Giertz, "Extremely Low Frequency Electromagnetic Energy in the Air," Journal of Atmospheric and Solar-Terrestrial Physics, Vol. 72, No. 9-10, 2010, pp. 767773. http://dx.doi.org/10.1016/j.jastp.2010.03.022

[14] A. Terras, "Fourier Analysis on Finite Groups and Applications," Cambridge University Press, Cambridge, 1999.

[15] B. I. Bleaney and B. Bleaney, "Electricity and Magnetism," Oxford University Press, Amen House, London, 1965.

[16] D. B. Melrose and R. C. McPhedran, "Electromagnetic Processes in Dispersive Media," Cambridge University Press, Cambridge, 1991.

[17] F. K. Kneubühl, "Oscillations and Waves," Springer, Berlin, 1997.

[18] R. A. Serway and J. W. Jewett, "Principles of Physics," 4th Edition, Cengage Learning, Stamford, 2005.

[19] L. A. Ostrovsky and A. I. Potapov, "Modulated Waves: Theory and Application," Johns Hopkins University Press, Baltimore, 2002. 